\title{
AGEs and renal sodium handling: association with hypertension
}

\author{
Daisuke Suzuki $^{1,2} \cdot$ Satoshi Hoshide ${ }^{1}$ Kazuomi Kario ${ }^{1}$
}

Received: 25 November 2021 / Revised: 8 December 2021 / Accepted: 8 December 2021 / Published online: 19 January 2022

(c) The Author(s), under exclusive licence to The Japanese Society of Hypertension 2022

Advanced glycation end products (AGEs) are a group of molecules produced by proteins, nucleic acids, and lipids through a nonenzymatic glycation process called the Maillard reaction [1]. AGEs can be classified into those of exogenous and endogenous origin, and exogenous AGEs are produced by a high-temperature diet [1]. On the other hand, the endogenous AGEs accumulate with age, oxidative stress, and chronic diseases such as hypertension, diabetes and chronic kidney disease [1]. Conversely, accumulation of AGEs can also cause or exacerbate disease, such as by increasing arterial stiffness or inducing cardiovascular events. However, this association may not be a direct causeand-effect relationship, and may be related to other pathways such as those involved in the generation of oxidative stress $[1,2]$.

While AGEs are known to be associated with hypertension and can cause atherosclerosis and cardiovascular disease through hypertension, the pathogenesis by which AGEs cause increased blood pressure and hypertension is not fully understood. One mechanism may be regulation via the kidneys. Accumulation of AGEs causes glomerular hypertrophy and injury, which results in proteinuria and eventual end-stage renal disease [1]. The kidneys may also play a role in the metabolism of AGEs, since AGEs have been shown to be absorbed and catabolized by the renal proximal tubule [2]. Furthermore, AGEs induce renal proximal cell apoptosis and cause inflammatory, thrombotic and fibrotic change [3]. The effect of these changes on renal sodium handling is unclear, but it is possible that they may lead to

\section{Kazuomi Kario}

kkario@jichi.ac.jp

1 Division of Cardiovascular Medicine, Department of Medicine, Jichi Medical University School of Medicine, 3311-159 Yakushiji, Shimotsuke-shi, Tochigi 329-0498, Japan

2 Department of Medicine, Division of Endocrinology and Metabolism, Jichi Medical University Saitama Medical Center, 1-847 Amanuma-cho, Omiya-ku, Saitama 330-8503, Japan some functional changes in the renal sodium handling, such as increase of renal sodium reabsorption.

Increase of renal sodium handling may be associated with high blood pressure and hypertension. Of the sodium ions filtered out of the glomerulus, $70-80 \%$ are reabsorbed by the proximal tubules; [4] therefore, it is possible that an increase in proximal sodium handling may be the main form of high blood pressure and hypertension related to sodium handling. Proximal sodium handling is often assessed by lithium ion handling, because lithium ions are exclusively absorbed in the proximal tubules using the same pathway as used for sodium-ion reabsorption. A previous report showed that an increase in baseline proximal fractional sodium reabsorption was associated with increases in systolic blood pressure (SBP) and diastolic blood pressure (DBP) after 8 years [5]. In addition, a 1 standard deviation increase in baseline proximal fractional sodium reabsorption was associated with development of hypertension after 8 years [odds ratio 1.34 (95\% CI 1.06-1.70)] [5]. Another study found that increased proximal sodium reabsorption was associated with increased arterial stiffness [6]. Therefore, proximal sodium reabsorption is one of the factors regulating blood pressure, and an increase in proximal sodium reabsorption may be associated with atherosclerosis.

Both AGEs and proximal sodium reabsorption are associated with hypertension and arterial stiffness, but the linkage between AGEs and proximal sodium reabsorption and the association between this linkage and hypertension are unknown. To fill this knowledge gap, a report by Huang et al. published in Hypertension Research investigated this association [7].

In their cross-sectional study, Huang and colleagues enrolled 989 patients who were suspected of having hypertension but who had not yet been treated, in order to investigate the association between AGEs and renal sodium handling as assessed by fractional excretions of lithium $\left(\mathrm{Fe}_{\mathrm{Li}}\right)$. In addition, they investigated the association between AGEs and change in blood pressure or development of hypertension classified by baseline sodium dietary intake assessed by $24 \mathrm{~h}$ urinary $\mathrm{NaCl}$ excretion. The results 
showed that the level of $\mathrm{Fe}_{\mathrm{Li}}$ in the patients in the top quintile of AGEs level was lower than that in patients in quintiles 1 to 4 (18.3\% vs. $21.6 \%)$. Only in patients with a baseline sodium dietary intake of $<6 \mathrm{~g}$ as assessed by $24 \mathrm{~h}$ urinary excretion of sodium, and not in those with baseline sodium dietary intake $>6 \mathrm{~g}$, the change of DBP in patients with the top quintile of AGEs levels was higher compared with that in patients in with quintiles 1-4 of AGEs levels. In addition, the risk of development of hypertension as defined by ambulatory blood pressure monitoring and recent initiation of antihypertensive drug treatments in the patients in the top AGEs quintile was higher than the risk in patients in AGEs quintiles 1-4 quintiles [hazard ratio: 3.73 (95\% CI 1.17-11.9)].

These results show that excess serum AGEs may be associated with increased renal sodium reabsorption. In addition, only in patients with low $24 \mathrm{~h}$ urine $\mathrm{NaCl}$ excretion was excess serum AGEs possibly associated with hypertension development. These results are interesting and may fill the knowledge gap in regard to the role played by AGEs in the development of hypertension, but in light of the study's several limitations, further interpretation of the findings may be needed.

First, the participants in the study by Huang et al.—even those with excess AGEs-had relatively low cardiovascular risk. The participants in this study were suspected of having hypertension and referred for ambulatory blood pressure monitoring but had not yet been untreated. However, the mean SBP and DBP were relatively low (even in the high AGEs group, these values were $132.8 \mathrm{mmHg}$ and 82.7 $\mathrm{mmHg}$ ), and the prevalence of clinical hypertension and ambulatory hypertension was $30.7 \%$ and $61.2 \%$ in the low AGEs group and $33.8 \%$ and $65.7 \%$ in the high AGEs group, respectively. In addition, the prevalence of coronary risk factors was relatively low, and even in the high AGEs group, the prevalence of diabetes was only 5.6\%. Thus, even the patients with excess AGEs in their study had a low risk of cardiovascular disease. The significance of excess AGEs in these patients is not yet known. In contrast, the results of this study showed that only in patients with low $24 \mathrm{~h}$ urine $\mathrm{NaCl}$ excretion, excess serum AGEs may be associated with the development of hypertension, and even in patients with a low risk of cardiovascular disease and low salt intake, excess AGEs may be a risk factor for the development of hypertension. This point is also important in terms of risk stratification of hypertension in this population.

Second, the participants were relatively young-indeed, only $20 \%$ of participants of this study were older than 60 years-and only increased DBP change, not SBP change, was observed. Clearly, both high SBP and high DBP are independent risk factors for cardiovascular events. SBP and DBP in older people may have different significance than those in younger people. For example, although SBP increases with age, DBP often decreases with age. The compliance of the large elastic arteries of older people was reduced by atherosclerosis [8]. This reduction in compliance of the large elastic arteries may be the reason for the relatively high proportion of isolated systolic hypertension in the elderly. Thus, the results of the study by Huang et al. may be applicable in young people but not in elderly people. In addition, the significance of isolated diastolic hypertension in young people is not yet known and needs to be investigated. Further research is also needed to determine whether the results are similar in the general population.

Third, why only patients with low $24 \mathrm{~h}$ urine $\mathrm{NaCl}$ excretion and excess serum AGEs were associated with the development of hypertension remains unknown. The authors stated that this may have been due to salt sensitivity. Salt sensitivity, which is often observed in older individuals and those with chronic kidney disease and metabolic syndrome [8], may be associated with renal sodium handling [9]. A previous study on patients with salt sensitivity showed that such patients had high proximal sodium handling on high salt but low proximal sodium handling on low salt [9]. In a related study, the authors found that an increase in DBP was associated with high proximal sodium handing but only in patients on a low salt diet [10]. The results of the study by Huang et al. may demonstrate that under high sodium conditions, the contribution of salt to blood pressure is large and significant, and therefore, the contribution of AGEs to blood pressure may be ignored. In contrast, at low sodium, the accumulation of AGEs may be a residual risk factor for hypertension [7].

Huang et al. showed the association between AGEs and renal sodium handling, and that between AGEs and development of hypertension. An excess of serum AGEs was shown to be associated with development of hypertension. This association may be involved with renal sodium handling, especially in patients with a low salt intake. Even in patients at low risk of cardiovascular disease, excess AGEs may lead to the development of hypertension through an increase in renal sodium handling, which may lead to cardiovascular disease.

\section{Compliance with ethical standard}

Conflict of interest The authors declare no competing interests.

Publisher's note Springer Nature remains neutral with regard to jurisdictional claims in published maps and institutional affiliations.

\section{References}

1. Zeng C, Li Y, Ma J, Niu L, Tay FR. Clinical/translational aspects of advanced glycation end-products. Trends Endocrinol Metab. 2019;30:959-73. 
2. Stinghen AE, Massy ZA, Vlassara H, Striker GE, Boullier A. Uremic toxicity of advanced glycation end products in CKD. J Am Soc Nephrol. 2016;27:354-70.

3. Matsui T, Yamagishi S, Takeuchi M, Ueda S, Fukami K, Okuda S. Irbesartan inhibits advanced glycation end product (AGE)induced proximal tubular cell injury in vitro by suppressing receptor for AGEs (RAGE) expression. Pharm Res. 2010;61:34-9.

4. Kleta R, Bockenhauer D. Salt-losing tubulopathies in children: what's new, what's controversial? J Am Soc Nephrol. 2018;29:727-39.

5. D'Elia L, Cappuccio FP, Iacone R, Russo O, Galletti F, Strazzullo P. Altered renal sodium handling and risk of incident hypertension: results of the Olivetti Heart Study. PLoS One. 2017;12: e0171973.

6. Cwynar M, Gasowski J, Gryglewska B, Gluszewska A, Kwater A, Krolczyk J, et al. Insulin resistance and renal sodium handling influence arterial stiffness in hypertensive patients with prevailing sodium intake. Am J Hypertens. 2019;32:848-57.

7. Huang QF, Cheng YB, Guo QH, Liu CY, Kang YY, Sheng CS, et al. Clinic and ambulatory blood pressure in relation to the interaction between plasma advanced glycation end products and sodium dietary intake and renal handling. Hypertens Res. 2021. https://doi.org/10.1038/s41440-021-00805-z.

8. Umemura S, Arima H, Arima S, Asayama K, Dohi Y, Hirooka Y, et al. The Japanese Society of Hypertension guidelines for the management of hypertension (JSH 2019). Hypertens Res. 2019;42:1235-481.

9. Chiolero A, Maillard M, Nussberger J, Brunner HR, Burnier M. Proximal sodium reabsorption: an independent determinant of blood pressure response to salt. Hypertension. 2000;36:631-7.

10. Zou J, Li Y, Yan CH, Wei FF, Zhang L, Wang JG. Blood pressure in relation to interactions between sodium dietary intake and renal handling. Hypertension. 2013;62:719-25. 